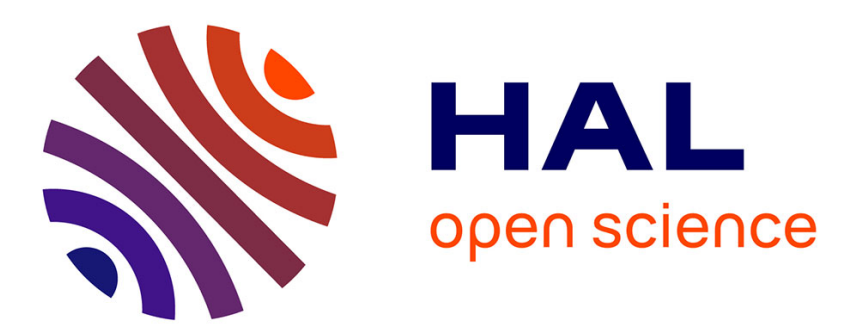

\title{
White and weighted averages over solutions of Thouless Anderson Palmer equations for the Sherrington Kirkpatrick spin glass
}

\author{
Cirano de Dominicis, M. Gabay, T. Garel, Henri Orland
}

\section{To cite this version:}

Cirano de Dominicis, M. Gabay, T. Garel, Henri Orland. White and weighted averages over solutions of Thouless Anderson Palmer equations for the Sherrington Kirkpatrick spin glass. Journal de Physique, 1980, 41 (9), pp.923-930. 10.1051/jphys:01980004109092300 . jpa-00208923

HAL Id: jpa-00208923

https://hal.science/jpa-00208923

Submitted on 1 Jan 1980

HAL is a multi-disciplinary open access archive for the deposit and dissemination of scientific research documents, whether they are published or not. The documents may come from teaching and research institutions in France or abroad, or from public or private research centers.
L'archive ouverte pluridisciplinaire HAL, est destinée au dépôt et à la diffusion de documents scientifiques de niveau recherche, publiés ou non, émanant des établissements d'enseignement et de recherche français ou étrangers, des laboratoires publics ou privés. 


\title{
LE JOURNAL DE PHYSIQUE
}

\section{White and weighted averages over solutions of Thouless Anderson Palmer equations for the Sherrington Kirkpatrick spin glass}

\author{
C. De Dominicis, M. Gabay (*), T. Garel (*) and H. Orland \\ Division de la Physique, Service de Physique Théorique, C.E.N. de Saclay, B.P. No 2, 91190 Gif sur Yvette, France
}

(Reçu le 19 mars 1980, accepté le 5 mai 1980)

\begin{abstract}
Résumé. - Nous étudions les valeurs moyennes d'observables prises sur l'ensemble des solutions des équations de Thouless Anderson Palmer. Ces moyennes sont calculées à poids constant (moyenne blanche) ou variable. Pour la moyenne blanche nous donnons des résultats analytiques près de la température de transition et près de $T=0$ en bon accord qualitatif avec les résultats attendus pour le paramètre d'ordre d'Edwards Anderson. La valeur trop élevée de l'énergie du fondamental est attribuée au caractère non discriminatoire de la moyenne blanche. Nous vérifions la stabilité locale dans toutes les directions pour le col pertinent (diagonal dans l'espace des répliques). Nous étudions aussi la moyenne microcanonique à $T=0$. Dans ce cas le col diagonal est instable et les équations pour le col non diagonal ne sont pas solubles par des moyens purement analytiques.
\end{abstract}

\begin{abstract}
We study white and weighted averages of observables over all solutions of Thouless Anderson and Palmer equations. For the white average we give analytic results near the transition temperature and near $T=0$, in good qualitative agreement with expected results for the Edwards Anderson order parameter. A too high value for the ground state energy is attributed to the non discriminating character of the white average. Local stability in all directions is verified for the relevant saddle point (diagonal in replica space). We also study a microcanonical average at $T=0$. In that case, the diagonal saddle point is unstable and equations for the off-diagonal one are not soluble by purely analytical means.
\end{abstract}

1. Introduction. - Finding a sensible mean field theory for spins glasses still remains an unsolved problem. The Edwards Anderson [E.A., 1] order parameter was used by Sherrington and Kirkpatrick [S.K., 2] in their long range random Ising model described by the Hamiltonian :

$$
\mathscr{H}=-\frac{1}{2} \sum_{j \neq l} J_{j l} \sigma_{j} \sigma_{l} \quad j, l=1,2, \ldots, N
$$

together with the probability law

$$
p\left(J_{j l}\right)=\left(\frac{N}{2 \pi J^{2}}\right)^{1 / 2} \exp -\frac{N J_{j l}^{2}}{2 J^{2}}
$$

for the random coupling $J_{j l}$. To calculate quenched averages, the S.K. solution relies on the use of the (symmetric) replica trick which turns out to be incorrect for $T<T_{\mathrm{c}}(\equiv J)$. Symmetry breaking schemes

$\left(^{*}\right)$ Laboratoire de Physique des Solides (L.A.2), Université Paris-Sud, 91405 Orsay, France. between replicas have mostly failed in their description of the low temperature phase, the most promising one being that of Parisi [3]. Following a different route Thouless Anderson and Palmer [T.A.P., 4] have written $N$ equations for the thermal averages

$$
\begin{gathered}
m_{j}=\left\langle\sigma_{j}\right\rangle_{T} \\
F_{j} \equiv \tanh ^{-1} m_{j}-\sum_{l} \frac{J_{j l} m_{l}}{T}+ \\
+m_{j} \sum_{l} \frac{J_{j l}^{2}}{T^{2}}\left(1-m_{l}^{2}\right)=0,
\end{gathered}
$$

equations that are valid to order $1 / N$. These authors have given an analytical solution for $T \leqslant T_{\mathrm{c}}$ and together with some assumptions, numerical results for $T \simeq 0$ in close agreement with Monte-Carlo computations.

Recently it has been realized [5] that computing averages (over the random bond distribution) of solutions $\left\{m_{j}\right\}$ of T.A.P. equations, can be reduced to a self consistent one site problem. The self consis- 
tent equations involve four order parameters. They contain as special cases the S.K. solution (where the E.A. order parameter is the only survivor) and the Sommers [6] solution (where the linear response anomaly also appears).

In the following we first recall, in section 2 , the reduction to a one site self consistent problem. In section 3 it is shown that the (averaged) number $\mathcal{N}_{\mathrm{s}}$ of solutions to T.A.P. equations is large. This forces us to decide what weight to attribute to each solution. Also in order to build averages over the ensemble of solutions (rather than sum over solutions) we are forced to introduce what we were striving to avoid i.e. replicas. In section 4 we argue to the relative «innocence " of our replicas and point to two types of saddle points (diagonal in replica space, and offdiagonal). In section 5 we concentrate on the diagonal saddle point of the white (equal weight) average over solutions. We give analytic results near $T=T_{\mathrm{c}}$ and $T=0$. These results are qualitatively good but too high for the ground state energy, presumably on account of the chosen weight. To proceed further we work in section 6 in the simpler limiting case $T=0$. We confirm the local stability of the white average diagonal saddle point. For the microcanonical average the diagonal saddle point is unstable. Equations for the off-diagonal saddle point - a more likely but tougher candidate - are exhibited in the appendix.

2. Transformation to a self consistent one site problem. - The generating functional of the $m_{j}$ correlation functions, can be written, à la Martin Siggia Rose [7] as :

$$
\hat{Z}\left\{l_{j}, J_{j l}\right\}=\int \prod_{j} \mathrm{~d} m_{j} \delta\left(F_{j}\right) \operatorname{det} \frac{\partial F_{j}}{\partial m_{l}} \exp \left(\sum_{k} l_{k} m_{k}\right),
$$

where the delta functions impose that T.A.P. equations (3) are satisfied and the determinant is the Jacobian normalizing properly the delta functions. We express the Jacobian $f$ as :

$$
\tilde{J} \equiv \operatorname{det} \frac{\partial F_{j}}{\partial m_{l}}=\int \prod_{j} \mathrm{~d} \eta_{j}^{*} \mathrm{~d} \eta_{j}^{*} \exp \sum_{j, l} \eta_{j}^{*}\left\{\left[\frac{1}{1-m_{j}^{2}}+\sum_{k} \frac{J_{j k}^{2}}{T^{2}}\left(1-m_{k}^{2}\right)\right] \delta_{j l}-\frac{J_{j l}}{T}-2 m_{j} \frac{J_{j l}^{2}}{T^{2}} m_{l}\right\} \eta_{l}
$$

where the integration is over anticommuting $c$-numbers $\eta, \eta^{*}$, and we also use :

$$
\delta\left(F_{j}\right)=\int \frac{\mathrm{d} \hat{m}_{j}}{2 \pi} \exp \left\{-i \hat{m}_{j}\left(\tanh ^{-1} m_{j}-\sum_{l} \frac{J_{j l}}{T} m_{j}+m_{j} \sum_{l} \frac{J_{j l}^{2}}{T^{2}}\left(1-m_{l}^{2}\right)\right)\right\} .
$$

On the properly normalized expression (4), we may now directly take quenched averages over the probability law (2), as

$$
\hat{Z}_{\mathrm{av}}\left\{l_{j}\right\}=\int \prod_{(j l)} p\left(J_{j l}\right) \hat{Z}\left\{l_{j} ; J_{j l}\right\}
$$

Following notations of reference [5], one then introduces four (order) parameters

$$
\begin{array}{r}
q=\frac{1}{N} \sum_{j} m_{j}^{2} \\
1-q+a \equiv g=\frac{1}{N} \sum_{j} i \hat{m}_{j} m_{j} \\
\hat{q}=\frac{1}{N} \sum_{j}\left(i \hat{m}_{j}\right)^{2} \\
1-q-b \equiv n=\frac{1}{N} \sum_{j} \eta_{j}^{*} \eta_{j}
\end{array}
$$

that turn out to decouple all sites, i.e. transform the problem into a self consistent one site problem. Here $q$ is the E.A. order parameter, $g$ the linear response, with its exhibited anomaly $a$, and $n$ the ghost occupation.

Indeed, if we impose constraints (8)-(11), the generating functional (7) reads :

$$
\hat{Z}_{\mathrm{av}}=\left(\frac{N}{2 \pi}\right)^{4} \int \prod_{v=1}^{4} \mathrm{~d} x_{v} \mathrm{~d} \tilde{x}_{v} \int \prod_{j} \mathrm{~d} m_{j} \frac{\mathrm{d} \hat{m}_{j}}{2 \pi} \mathrm{d} \eta_{j} \mathrm{~d} \eta_{j}^{*} \exp \left\{N C+\sum_{j} L_{j}+\sum_{j l} \eta_{j}^{*} M_{j l} \eta_{l}\right\}
$$

where we use $x_{v}$ for $q, g, \hat{q}, n$ and $\tilde{x}_{v}$ for the corresponding constraint variables. Here :

$$
C=-\frac{J^{2}}{2 T^{2}}\left[-g^{2}+n^{2}-q \hat{q}-2(1-q)(n-g)\right]-i \sum_{v} x_{v} \tilde{x}_{v}
$$


and

$$
L_{j}=-i \hat{m}_{j} \tanh ^{-1} m_{j}+i \tilde{q} m_{j}^{2}+i \tilde{g}\left(i \hat{m}_{j} m_{j}\right)+i \tilde{\hat{q}}\left(i \hat{m}_{j}\right)^{2}+l_{j} m_{j}
$$

$$
M_{j l}=\left(i \tilde{n}+\frac{1}{1-m_{j}^{2}}\right) \delta_{j l}-\frac{J^{2}}{2 T^{2}}\left[2 m_{j} m_{l}+i \hat{m}_{j} m_{l}+i \hat{m}_{l} m_{j}\right]
$$

The only non locality left is now removed by integration over fermionic variables that replaces the integrand in (12) by exp $N \mathcal{A}$ (within a factor of order one)

$$
\mathcal{A}=C+\ln \int \mathrm{d} m \frac{\mathrm{d} \hat{m}}{2 \pi} \mathrm{e}^{L}\left(i \tilde{n}+\frac{1}{1-m^{2}}\right) .
$$

Saddle point conditions on $\mathcal{A}$ then determine $q, g$, $\hat{q}, n$ self consistently as :

$$
\begin{gathered}
q=\mathcal{N}^{-1} \int_{-1}^{+1} \mathrm{~d} m \mathrm{e}^{\mathfrak{L}}\left(B+\frac{1}{1-m^{2}}\right) m^{2} \\
g=\mathcal{N}^{-1} \int_{-1}^{+1} \mathrm{~d} m \mathrm{e}^{\mathrm{f}}\left(B+\frac{1}{1-m^{2}}\right) \times \\
\times\left(\frac{\tanh ^{-1} m-A m}{Q}\right) m \\
\hat{q}=\mathcal{N}^{-1} \int_{-1}^{+1} \mathrm{~d} m \mathrm{e}^{\mathfrak{f}\left(B+\frac{1}{1-m^{2}}\right) \times} \\
n=\mathcal{N}^{-1} \int_{-1}^{+1} \mathrm{~d} m \mathrm{e}^{\mathfrak{L}}
\end{gathered}
$$

together with the normalization

$$
\mathcal{N}=\int_{-1}^{+1} \mathrm{~d} m \mathrm{e}^{\mathfrak{L}}\left(B+\frac{1}{1-m^{2}}\right)
$$

and

$$
\begin{aligned}
\mathcal{L}=-\frac{1}{2 Q}\left(\tanh ^{-1} m-\right. & A m)^{2}+ \\
& +\left(\frac{\hat{Q}}{2}+A+B\right) m^{2} .
\end{aligned}
$$

The notation used is $X_{v} \equiv\left(J^{2} / T^{2}\right) x_{v}$. These self consistent equations incorporate the S.K. solution $(\hat{q}=a=b=0)$ and the Sommers [6] solution $(\hat{q}=a+b=0)$ as special cases.

3. Average number of solutions and normalization. So far we have assumed that there exists only one solution (beside the trivial one $m_{j}=0$ ) to T.A.P. equations. This applies to solutions that can be retrieved by perturbation resummation as S.K. or Sommers solutions and can be checked with the help of equation (12) since the number of solutions $\mathcal{N}_{\mathrm{s}}$, averaged, is given by

$$
\overline{\mathcal{N}_{\mathrm{s}}}=\hat{Z}_{\mathrm{av}}(l=0)
$$

For the general solution (17)-(22) however this number turns out to be of order $\exp N \mathcal{A}_{\mathrm{c}}(T)$, where $\mathcal{A}_{\mathrm{c}}(T)$ vanishes near $T_{\mathrm{c}}$.

This immediately raises one technical question namely that the Jacobian $\gamma$ defined in equation (5) should be replaced by $|g|$ in equation (4), this replacement being irrelevant when the solution to T.A.P. equations is unique. Again we have checked that $g$ keeps the same sign near $T_{\mathrm{c}}$ and at $T=0$ (see also comment in conclusion).

Moreover, the existence of many solutions raises conceptual questions :

1) Since equation (4) sums over solutions, when we have many of them, we have to give a weight $\mathcal{T}\left\{m_{s}\right\}$ to each solutions. In the following we consider an uniform weight (white average) and a weight that selects solutions of a given energy (microcanonical weight).

2) When we have a unique solutions $\left\{m_{j, s}\right\}$, the value of any observable $\left\langle 0\left(m_{j}\right)\right\rangle$ is given by $0\left(m_{j, s}\right)$. When we have many, we have to normalize the sum over solutions $s$ as :

$$
\left\langle 0\left(m_{j}\right)\right\rangle=\frac{\sum_{s} 0\left(m_{j, s}\right) \mathfrak{T}\left\{m_{s}\right\}}{\sum_{s} \mathfrak{T}\left\{m_{s}\right\}} .
$$

This necessity seems to push us again towards the technique we wanted to avoid, namely replicas.

4. Introducing « innocent » replicas. - To be able to take a quenched average of (24) over the bond probability law (2), we use replicas and write :

$$
\begin{aligned}
\overline{\left\langle 0\left(m_{j}\right)\right\rangle}=\frac{\overline{\sum_{s} 0\left(m_{j, s}\right) \mathfrak{T}\left\{m_{s}\right\}}}{\sum_{s} \mathfrak{T}\left\{m_{s}\right\}}= \\
=\lim _{n \rightarrow 0} \int_{(j l)} \mathrm{d} J_{j l} p\left(J_{j l}\right) \int \prod_{j, \alpha} \mathrm{d} m_{j}^{\alpha} \delta\left(F_{j}^{\alpha}\right) \times \\
\quad \times \operatorname{det}\left(\frac{\partial F_{j}^{\alpha}}{\partial m_{k}^{\alpha}}\right) O\left(m_{j}^{\alpha}\right) \mathfrak{T}\left\{m_{j}^{\alpha}\right\} .
\end{aligned}
$$

The replica index $\alpha$ runs from one to $n$, and $\alpha_{0}$ is fixed.

Note that our replicas help to normalize with respect to the sum over solutions of T.A.P. equations, in contradistinction with usual replicas which help in normalizing with respect to the sum over all configurations. When only one solution exists the replica way and the no replica way give identical 
results. In that sense our replicas should be rather « innocent " $\left({ }^{1}\right)$.

The averaged order parameters (or correlations) can now be « diagonal "

$$
\begin{aligned}
q^{\alpha_{0} \alpha_{0}}=\frac{1}{N} \sum_{j} \overline{\left(m_{j}^{\alpha_{0}}\right)^{2}}= \\
\quad=\frac{1}{N} \sum_{j} \overline{\sum_{s} m_{j, s}^{2} \mathfrak{T}\left\{m_{s}\right\} / \sum_{s} \mathfrak{T}\left\{m_{s}\right\}}
\end{aligned}
$$

or « off-diagonal "

$$
\begin{aligned}
q^{\alpha_{0} \beta_{0}}= & \frac{1}{N} \sum_{j} \overline{m_{j}^{\alpha_{0}} m_{j}^{\beta_{0}}}= \\
& =\frac{1}{N} \sum_{j} \overline{\left(\sum_{s} m_{j, s} \mathfrak{T}\left\{m_{s}\right\} / \sum_{s} \mathfrak{T}\left\{m_{s}\right\}\right)^{2}}
\end{aligned}
$$

where it is understood that the $n=0$ limit is taken. Note that $q^{\alpha_{0} \alpha_{0}} \geqslant q^{\alpha_{0} \beta_{0}}$.

The self consistent saddle point equations that generalize (17)-(22) are immediately written following the derivation of section 2 . Two distinct simple classes of solutions emerge from these equations :

1) A diagonal solution where :

$$
\begin{aligned}
& x^{\alpha \alpha}=x \\
& x^{\alpha \beta}=0 \quad \alpha \neq \beta
\end{aligned}
$$

with $x=q, \hat{q}, g, n$.

2) A solution with off-diagonal components :

$$
\begin{aligned}
& x^{\alpha \alpha}=x \\
& x^{\alpha \beta}=x_{0} \quad \alpha \neq \beta .
\end{aligned}
$$

We first specialize in the study of the diagonal solution for the white average case (eqs. (17)-(22)).

5. Analytic results for the white average diagonal solution. - Given the large number of solutions that seem to exist to T.A.P. equations, the average $\sum_{s}\left(m_{j, s}\right) / \sum_{s}$ appearing in (27) seems likely to be very small, an indication that the diagonal solution (rather than the off-diagonal) will emerge from the dominant saddle point. Thus it is reasonable to study (17)-(22) and we now quote a few results concerning solutions thereof.

First we note that there are two classes of solutions ( $B=0$ and $B \neq 0$ ) since equation (20) implies :

$B\left[\int_{-1}^{+1} \mathrm{~d} m\left\{B+\frac{1}{1-m^{2}}-\frac{J^{2}}{T^{2}}\left(1-m^{2}\right)\right\} \mathrm{e}^{\mathfrak{L}}\right]=0$.

( $\left.{ }^{1}\right)$ Indeed the Sommers solution for example is obtained in the direct approach of S.K. via a peculiar way of breaking the replica symmetry $[9,10]$. Here instead, it is obtained as a special case of the no replica way. It also arises as a special case of the diagonal saddle point in the replica way, see below.
The vanishing of the bracket together with $B=0$ is the Bray-Moore [8] criterion for the existence of a massless mode in the spin glass phase. Both $B=0$ and $B \neq 0$ give consistent results near $T_{c}$, but only $B=0$ turns out to be acceptable near $T=0$.

i) Near $T_{\mathrm{c}}$ : Setting $t=\left(T_{\mathrm{c}}-T\right) / T_{\mathrm{c}}$ from equations (8)-(10) we get, after some labour :

$$
\begin{aligned}
& q=t+t^{2}-\frac{5}{9} t^{3}+O\left(t^{4}\right) \\
& g=\frac{2}{3} t^{2}-\frac{2}{9} t^{3}+0\left(t^{4}\right) \\
& \hat{q}=-\frac{4}{9} t^{3}+\frac{4}{9} t^{4}+O\left(t^{5}\right)
\end{aligned}
$$

noting that we need $\hat{q}$ to order $t^{4}$ to obtain $(q, g)$ to order $t^{3}$. This value of $q$ leaves us with a stable solution in the sense of Bray-Moore [8] (mass of replicon positive and of order $t^{3}$ ).

ii) Near $T=0$ : The averaged internal energy $U$ is given by

$$
\bar{U}=-T^{2} \overline{\frac{\partial}{\partial T} \frac{F}{T}}
$$

where

$$
\begin{aligned}
-\frac{F}{T}=\sum_{(j l)} \frac{J_{j l}}{T} m_{j} m_{l} & +\frac{1}{2} \sum_{(j l)} \frac{J_{j l}}{T^{2}}\left(1-m_{j}^{2}\right)\left(1-m_{l}^{2}\right)- \\
& -\frac{1}{2} \sum_{j}\left[\left(1+m_{j}\right) \ln \frac{\left(1+m_{j}\right)}{2}\right. \\
& \left.+\left(1-m_{j}\right) \ln \frac{\left(1-m_{j}\right)}{2}\right]
\end{aligned}
$$

Near $T=0$,

$$
\varepsilon=\frac{\bar{U}}{N J}=-\frac{1}{2 T}\left[1-q^{2}+2 a q\right]
$$

and

$$
\begin{aligned}
& q=1-\frac{1}{2} T^{2} \\
& a=|\varepsilon| T
\end{aligned}
$$

where

$$
\begin{aligned}
|\varepsilon| & =\frac{2}{\sqrt{2 \pi}} \exp \left(-\frac{\varepsilon^{2}}{2}\right) \times \\
& \times\left[1+\frac{2}{\sqrt{2 \pi}} \int_{0}^{|\varepsilon|} \mathrm{e}^{-t^{2} / 2} \mathrm{~d} t\right]^{-1}=0.506
\end{aligned}
$$

The behaviour obtained near $T_{\mathrm{c}}$ and $T=0$ is in good qualitative agreement with results of reference $[4,8]$, equation (36) implying an entropy in $T^{2}$. However the numbers we obtain disagree with Monte-Carlo simulations $[11,12]$, in particular our value (38) for the ground state energy is too high. A plausible explanation for this result, stems from the type of unweighted (white) average that we are using. In the next section we consider a weight that selects the lowest ground state solution (paramagnetic solution $\left\{m_{j}=0\right\}$ excepted). To keep things simple we restrict ourselves to the zero temperature case. 
6. White and weighted averages at $\boldsymbol{T}=\mathbf{0}$. - At We have used the fact that :

zêro temperature equation (3) is replaced by :

$$
\varphi_{j}-\sum_{l} \frac{J_{j l}}{J} \operatorname{sgn} \varphi_{l}=0
$$

where $\operatorname{sgn} \varphi$ is sign of $\varphi$, and we have set :

$$
m_{j}=\tanh \frac{J \varphi_{j}}{T}
$$

$$
\sum_{l} \frac{J_{j l}^{2}}{T}\left(1-m_{l}^{2}\right) \sim \frac{J^{2}}{T}(1-q)
$$

is negligible as $T \rightarrow 0$ (an approximation that is not valid for the S.K. solution). Careful examination of the $T=0$ limit of equation (20) shows that it is consistent to take the Jacobian as unity (and neglect what would result from the local field term of (39) in this limit).

1) White average : We now have, instead of (7)

$$
\hat{Z}_{\mathrm{av}}^{0}\left(l_{j}\right)=\int \prod_{(j l)} \mathrm{d} J_{j l} p\left(J_{j l}\right) \int \prod_{j} \mathrm{~d} \varphi_{j} \delta\left(\varphi_{j}-\sum_{l} \frac{J_{j l}}{J} \operatorname{sgn} \varphi_{l}\right) \exp \left(\sum_{k} l_{k} \varphi_{k}\right)
$$

We introduce replicas as in (24), and instead of (8-11), (26), (27) we use the order parameters

$$
\begin{gathered}
q^{\alpha \beta}=\frac{1}{N} \sum_{j} \operatorname{sgn} \varphi_{j}^{\alpha} \varphi_{j}^{\beta} \quad \alpha \neq \beta \\
-i \frac{J}{T} g^{\alpha \beta} \equiv \gamma^{\alpha \beta}=\frac{1}{2 N} \sum_{j}\left(\hat{\varphi}_{j}^{\alpha} \operatorname{sgn} \varphi_{j}^{\beta}+\hat{\varphi}_{j}^{\beta} \operatorname{sgn} \varphi_{j}^{\alpha}\right) \\
-\frac{J}{T^{2}} \hat{q}^{\alpha \beta} \equiv \kappa^{\alpha \beta}=\frac{1}{N} \sum_{j} \hat{\varphi}^{\alpha} \hat{\varphi}^{\beta} \quad \alpha \neq \beta
\end{gathered}
$$

where the $T^{-1}, T^{-2}$ weights arise from $\frac{J}{T} \hat{m}=\varphi$.

$\bar{T}$ The only surviving diagonal order parameter is $\gamma^{\alpha x}$. The saddle point exponent $\mathcal{A}$ of equation (16) now reads :

$$
\begin{aligned}
t=-\frac{1}{2} \sum_{\alpha}\left(\gamma^{\alpha \alpha}\right)^{2}-\frac{1}{2} \sum_{\alpha \neq \beta}\left(\left(\gamma^{\alpha \beta}\right)^{2}+\kappa^{\alpha \beta} q^{\alpha \beta}\right) & -i \sum_{j} \gamma^{\alpha \alpha} \tilde{\gamma}^{\alpha \alpha}+ \\
& +\sum_{\alpha \neq \beta}\left(\gamma^{\alpha \beta} \tilde{\gamma}^{\alpha \beta}+\kappa^{\alpha \beta} \tilde{\kappa}^{\alpha \beta}+q^{\alpha \beta} \tilde{q}^{\alpha \beta}\right)+\ln \int \prod_{\alpha} \mathrm{d} \varphi^{\alpha} \frac{\mathrm{d} \hat{\varphi}^{\alpha}}{2 \pi} \exp \mathfrak{L} \\
\mathfrak{L}=-\sum_{\alpha}\left[i \hat{\varphi}^{\alpha} \varphi^{\alpha}+\frac{1}{2}\left(\hat{\varphi}^{\alpha}\right)^{2}\right] & +\sum_{\alpha} i \hat{\gamma}^{\alpha x} \hat{\varphi}^{\alpha} \operatorname{sgn} \varphi^{\alpha}+ \\
& +\sum_{\alpha \neq \beta}\left(i \hat{\gamma}^{\alpha \beta} \frac{1}{2}\left[\hat{\varphi}^{\alpha} \operatorname{sgn} \varphi^{\beta}+\hat{\varphi}^{\beta} \operatorname{sgn} \varphi^{\alpha}\right]+i \tilde{\kappa}^{\alpha \beta} \hat{\varphi}^{\alpha} \hat{\varphi}^{\beta}+i \tilde{q}^{\alpha \beta} \operatorname{sgn} \varphi^{\alpha} \operatorname{sgn} \varphi^{\beta}\right) .
\end{aligned}
$$

At the diagonal saddle point $\left(x^{\alpha \beta}=0, \tilde{x}^{\alpha \beta}=0, \alpha \neq \beta\right)$ one obtains :

$$
\tilde{\gamma}=\frac{2}{\sqrt{2 \pi}} \mathrm{e}^{-\tilde{i}^{2} / 2}\left[1+\frac{2}{\sqrt{2 \pi}} \int_{0}^{\tilde{\gamma}} \mathrm{e}^{-t^{2} / 2} \mathrm{~d} t\right]^{-1}=0.506
$$

This is also the ground state energy value in units of $J$. The saddle point value of $A$ that measures $\frac{1}{N} \ln \overline{\mathcal{N}}_{\mathrm{s}}$ is

$$
\mathcal{A}_{\mathrm{c}}(T=0)=-\frac{1}{2}(\tilde{\gamma})^{2}+\ln \left[1+\frac{2}{\sqrt{2 \pi}} \int_{0}^{\tilde{\gamma}} \mathrm{e}^{-t^{2} / 2} \mathrm{~d} t\right] \simeq 0.198
$$

(and $\mathcal{A}_{\mathrm{c}}(T=0)=0$ for S.K. and Sommers solutions).

Fluctuations about this saddle point are governed the matrix of the quadratic form in $\delta x^{\alpha \beta}, \delta \tilde{x}^{\alpha \beta}$. This matrix contains $n$ diagonal $2 \times 2$ blocks in $\delta \gamma^{\alpha x} \delta \tilde{\gamma}^{\alpha x}$

$$
\left|\begin{array}{ll}
-1 & -i \\
-i & -2 \tilde{\gamma}^{2}
\end{array}\right|
$$


and $n(n-1)$ off-diagonal $6 \times 6$ blocks in $\delta x^{\alpha \beta} \delta x^{\alpha \beta}$ that decompose into a $\delta x, \delta x$ block (in order $\left.\delta \gamma, \delta q, \delta \kappa\right)$,

$$
\left|\begin{array}{rrr}
-1 & 0 & 0 \\
0 & 0 & -\frac{1}{2} \\
0 & -\frac{1}{2} & 0
\end{array}\right|
$$

a fluctuation $\delta \tilde{x} \delta \tilde{x}$ block

$$
\left|\begin{array}{ccc}
-1 & i \tilde{\gamma} & i \tilde{\gamma}^{3} \\
i \tilde{\gamma} & -1 & \tilde{\gamma} \\
i \tilde{\gamma} & \tilde{\gamma}^{2} & -\tilde{\gamma}^{4}
\end{array}\right|
$$

the $\delta x \delta \tilde{x}$ blocks being $i \uparrow$. One easily checks that for the saddle point value given by (47) the quadratic form is (locally) stable.

The white average thus seems to give consistent results and the stability of the diagonal saddle point justifies the presumed « innocence » of our replicas.

A corresponding computation done for S.K. or Sommers solution would display their unstability.

2) Microcanonical or canonical average : given the large (averaged) number of T.A.P. solutions, we may wish to select the ones that are of lowest energy. This may be done by imposing a canonical weight $\exp -\left(F-F_{0}\right) / T$ or with equivalent results a microcanonical weight $\delta\left(-E+E_{0}\right)$. Here for example, $E_{0}$ will be determined to as to select the least degenerate (or lowest energy) solution, i.e. by imposing the condition

$$
\frac{1}{N} \ln \overline{\mathscr{N}}_{\mathrm{s}}=\mathcal{A}_{\mathrm{c}}\left(T=0, \varepsilon_{0}\right)=0 \text {. }
$$

The saddle point exponent becomes now

$$
\begin{array}{r}
\mathcal{t}=-\frac{1}{2} \sum_{\alpha}\left[\gamma^{\alpha \alpha} \gamma^{\alpha \alpha}+\frac{1}{2} \hat{\lambda}^{\alpha} \hat{\lambda}^{\alpha}+2 \lambda^{\alpha} \gamma^{\alpha x}\right]-\frac{1}{2} \sum_{\alpha \neq \beta}\left[\gamma^{\alpha \beta} \gamma^{\alpha \beta}+\kappa^{\alpha \beta} q^{\alpha \beta}+\frac{1}{2} \hat{\lambda}^{\alpha} \lambda^{\beta}\left(q^{\alpha \beta}\right)^{2}+\left(\lambda^{\alpha}+\lambda^{\beta}\right) \gamma^{\alpha \beta} q^{\alpha \beta}\right]+ \\
+i \hat{\lambda}^{\alpha} \varepsilon_{0}-i \sum_{\alpha, \beta}\left[\gamma^{\alpha \alpha} \tilde{\gamma}^{\alpha \alpha}+\gamma^{\alpha \beta} \tilde{\gamma}^{\alpha \beta}+\kappa^{\alpha \beta} \tilde{\kappa}^{\alpha \beta}+q^{\alpha \beta} \tilde{q}^{\alpha \beta}\right]+\ln \int \prod_{\alpha} \mathrm{d} \varphi^{\alpha} \frac{\mathrm{d} \varphi^{\alpha}}{2 \pi} \exp \mathcal{L}
\end{array}
$$

where $L$ is again given by (46).

The diagonal saddle point leads, after elimination of $\lambda$ and $\gamma$ to

$$
2\left|\varepsilon_{0}\right|-\tilde{\gamma}=\frac{2}{\sqrt{2 \pi}} \mathrm{e}^{-\tilde{\gamma}^{2} / 2}\left[1+\frac{2}{\sqrt{2 \pi}} \int_{0}^{\tilde{\gamma}} \mathrm{e}^{-t^{2} / 2} \mathrm{~d} t\right]
$$

and for condition (50), to

$$
0=\mathfrak{t}_{\mathrm{c}}\left(T=0, \varepsilon_{0}\right)=-\varepsilon_{0}^{2}+\frac{1}{2}\left(2\left|\varepsilon_{0}\right|-\tilde{\gamma}\right)^{2}+\ln \left[1+\frac{2}{\sqrt{2 \pi}} \int_{0}^{\tilde{\gamma}} \mathrm{e}^{-t^{2} / 2} \mathrm{~d} t\right] .
$$

This yields a ground state value

$$
\varepsilon_{0}=-0.791 \text {. }
$$

Note that equations (52)-(53) also admit the higher solution $\varepsilon_{0}=-0.286$.

However, as expected from turning around the argument mentioned above (Section 4), the diagonal saddle point should not be stable for the microcanonical ensemble. Indeed fluctuations computed as in (6 (1)) for the white average, show its instability.

The off-diagonal saddle point becomes obviously relevant if one tries to impose the condition

$$
\overline{\ln \mathcal{N}_{\mathrm{s}}} \sim 0(1)
$$

a more natural constraint than the above $\ln \overline{\mathcal{N}}_{\mathrm{s}} \sim 0(1)$. The self consistent saddle point equations can no longer be solved analytically (near $T=T_{\mathrm{c}}$ or $T=0$ ). One is left with four self consistent equations for four order parameter $\gamma, \gamma_{0} \kappa_{0}$ and $q_{0}$ (and the equation $\mathcal{A}_{\mathrm{c}}\left(\varepsilon_{0}\right)=0$ ). In these equations the order parameters appear as averages of "local " order parameters $\gamma(x, y), \gamma_{0}(x, y)$, etc... in external fields $x, y$, the averages over $x, y$, being taken with gaussian weights. These equations are given in the appendix. We do not yet know what ground state energy these equations imply.

7. Conclusion. - We have been able to reduce 
white and weighted averages over solutions of T.A.P. equations to a set of self consistent equations involving a small number of " order parameters".

We have studied extensively the "white" average and obtained a satisfactory behaviour near $T_{\mathrm{c}}$ (where solutions to T.A.P. equations are unique to order $t^{3}$ ) and a good qualitative behaviour near $T=0$ (where the average number of solutions is of order exp $0.2 \mathrm{~N}$ ). The stability of such an average has been verified at $T=0$, lending credence to the presumed innocence of our replica scheme.

We have also studied a "weighted " average, where one clearly sees the necessity to consider the offdiagonal saddle point (at $T=0$, and in contrast to the white average).

Because of their complexity we have not yet been able to reach any numerical answer concerning the self consistent equations arising at the off-diagonal saddle points. Besides, it is not clear whether, given a weighted average, the diagonal or off-diagonal saddle points dominate over the entire range of temperature between $T=0$ and $T=T_{\mathrm{c}}$, or whether one will undergo a cross over.

Of course, none of the weights mentioned are really "natural ». The only weight that may deserve to be so called, is the one that the S.K. model itself dictates. This study is differed to a further publication.

At the les Houches meeting (February 18-29th 1980) where this work was presented, we learned of the work of A.J. Bray and M. A. Moore who have followed a close but slightly different approach. They have obtained results for the microcanonical average at $T=0$ identical to equation (54). This ensemble seems to have been also considered by F. Tanaka and S. F. Edwards [13]. A. J. Bray and M. A. Moore have further studied numerically the region near $T=0$ where they show that the microcanonical average diagonal saddle point leads to a negative entropy. This last result fits with the instability revealed by the fluctuation matrix as mentioned above. Equations produced by M. A. Moore for the off-diagonal saddle point, seem to differ from the ones of the appendix (by involving more integrations). Finally their numerical work also confirms that the Jacobian does not change sign at least near $T=0$.

Acknowledgments. - One of us (C.D.) would like to thank E. Brezin for useful discussions.

\section{APPENDIX}

Microcanonical average at $T=0$ : off-diagonal saddle point equations. - We consider the off-diagonal saddle point for the microcanonical average at $T=0$ introduced in section $(6(2))$.

The order parameters involved are $\gamma \equiv \gamma^{\alpha x}, \gamma_{0} \equiv \gamma^{\alpha \beta}$, $\kappa_{0} \equiv \kappa^{\alpha \beta}, q_{0} \equiv q^{\alpha \beta}$ and the corresponding constraint variables $\tilde{\gamma}, \tilde{\gamma}_{0}, \tilde{\kappa}_{0}, \tilde{q}_{0}$. Besides, one has the constraint $\hat{\lambda}^{x} \equiv \lambda$ conjugate to $E-E_{0}$, and the condition on $E_{0}$, i.e. $n^{-1} A_{\mathrm{c}}\left(\varepsilon_{0}\right)=0$ that imposes minimal degeneracy for the projected states.

From expression $\mathfrak{A}$ given by equation (51), one writes saddle point equations. To work with real quantities only we use

$$
\begin{aligned}
g & =i \gamma \\
g_{0} & =i \gamma_{0} \\
l & =i \lambda
\end{aligned}
$$

(there should be no confusion with the corresponding quantities $\left(g, g_{0}\right)$ at $T \neq 0$ which contain an extra $\tau / J$ factor $)$. We now quote the result.

The order parameters are obtained in terms of double averages, e.g.

$$
\begin{aligned}
\left\langle\left\langle\hat{\varphi}^{2}\right\rangle\right\rangle= & \int_{-\infty}^{+\infty} \int_{-\infty}^{+\infty} \frac{\mathrm{d} x \mathrm{~d} y}{2 \pi} \times \\
& \times \exp \left(-\frac{1}{2}\left(x^{2}+y^{2}\right)\right)\left\langle\hat{\varphi}^{2}\right\rangle_{x . y}
\end{aligned}
$$

where

$$
\begin{aligned}
\left\langle\hat{\varphi}^{2}\right\rangle_{x, y} & =\Lambda^{\prime-1} \int_{-\infty}^{+\infty} \mathrm{d} \varphi \int_{-\infty}^{+\infty} \frac{\mathrm{d} \hat{\varphi}}{2 \pi} \hat{\varphi}^{2} \mathrm{e}^{\mathfrak{L}} \\
\Lambda^{\prime} & =\int_{-\infty}^{+\infty} \mathrm{d} \varphi \int_{-\infty}^{+\infty} \frac{\mathrm{d} \hat{\varphi}}{2 \pi} \mathrm{e}^{\mathfrak{L}} .
\end{aligned}
$$

The effective Lagrangian $[$ writes

$$
\begin{aligned}
& \mathcal{L}=-i \hat{\varphi} \varphi-\frac{1}{2}\left(1-q_{0}\right) \hat{\varphi}^{2}+ \\
& \quad+\left[g-g_{0}+l\left(1-q_{0}\right)\right] i \hat{\varphi} \operatorname{sgn} \varphi \\
& +B^{1 / 2} \operatorname{sgn} \varphi+A^{1 / 2} x i \hat{\varphi}+\left[q_{0}-A\right]^{1 / 2} y i \hat{\varphi}
\end{aligned}
$$

with

$$
\begin{aligned}
& B \equiv 2 i \tilde{q}_{0}=l^{2} q_{0}+2 l g_{0}-\kappa_{0} \\
& A \equiv \frac{\tilde{\gamma}_{0}^{2}}{2 i \tilde{q}_{0}}=\frac{\left(g_{0}+l q_{0}\right)^{2}}{l^{2} q_{0}+2 l g_{0}-\kappa_{0}} .
\end{aligned}
$$

Note that the gaussian $\hat{\varphi}$ integration is trivially effected. The $\varphi$ integration leads to error functions. For simplicity we do not carry them out here. Note also that $x$ and $y$ play the role of external fields.

Given those definitions we write now the four equations determining $g, g_{0}, \kappa_{0}, q_{0}$ :

$$
\begin{aligned}
g & =\langle i \hat{\varphi} \operatorname{sgn} \varphi\rangle \\
g-g_{0} & =\left[\left\langle\langle x i \hat{\varphi}\rangle-\left(\frac{A}{q_{0}-A}\right)^{1 / 2}\langle\langle y i \hat{\varphi}\rangle]\right]\right. \\
1-q_{0} & =B^{-1 / 2}\left[\langle x \operatorname{sgn} \varphi\rangle-A^{1 / 2}\left(g-g_{0}\right)\right] \\
\kappa_{0} & =\left\langle\left\langle\hat{\varphi}^{2}\right\rangle+\left(q_{0}-A\right)^{-1 / 2}\langle y i \hat{\varphi}\rangle .\right.
\end{aligned}
$$


Equations determining $l$ and $\varepsilon_{0}$ (saddle point $\lambda$ and minimal degeneracy) write

$$
\begin{array}{r}
\left|\varepsilon_{0}\right|=\frac{l}{2}\left(1-q_{0}^{2}\right)+g-g_{0} q_{0} \\
l\left|\varepsilon_{0}\right|=\frac{l^{2}}{4}\left(1-q_{0}\right)^{2}-\frac{1}{2}\left(g^{2}-g_{0}^{2}\right)-l g_{0}\left(1-q_{0}\right)+ \\
+\frac{\kappa_{0}}{2}\left(1-q_{0}\right)+\ln \text {. }
\end{array}
$$

This completes the set of self consistent equations. These equations, via double averages defined in (A.4-A.6), involve at most, double gaussian integrals over ratios involving gaussians or error-functions.

\section{References}

[1] Edwards, S. F., ANderson, P. W., J. Phys. F 5 (1975) 965.

[2] Sherrington, D., Kirkpatrick, S., Phys. Rev. Lett. 35 (1975) 1792.

[3] Parisi, G., Phys. Rev. Lett. 43 (1979) 1754.

[4] Thouless, D. J., Anderson, P. W., Palmer, R. G., Philos. Mag. 35 (1977) 593.

[5] De Dominicis, C., C.R. Hebd. Séan. Acad. Sci., Paris B 289 (1979) 281

[6] Sommers, H. J., Z. Phys. B 31 (1978) 301.
[7] Martin, P. C., Siggia, E., Rose, H., Phys. Rev. A 8 (1973) 423.

[8] Bray, A. J., Moore, M. A., J. Phys. C 12 (1979) L-441.

[9] Bray, A. J., Moore, M. A., J. Phys. C 13 (1980) 419.

[10] De Dominicis, C., Garel, T., J. Physique Lett. 40 (1979) L-575.

[11] Kirkpatrick, S., Sherrington, D., Phys. Rev. B 17 (1978) 4384.

[12] Palmer, R. G., Pond, C. M., J. Phys. F 9 (1979) 1451.

[13] Tanaka, F. and Edwards, S. F., Preprint 1980. 